\title{
A multimedia introduction to chaos
}

\author{
Yih-Yuh Chen \\ Physics Department, National Taiwan University, Taipei, Taiwan, ROC
}

\begin{abstract}
With the advent of truly fast, but relatively inexpensive, personal computers, one can easily perform a desktop numerical experiment for some of the simplest chaotic dynamical systems, and view the animated dynamics "on-the-fly". When incorporated with sound effects, learning the basic ideas behind the seemingly complex behavior this way can be an enjoyable experience. Here, we will give a poor man's version of how multimedia can aid in one's understanding of this exploding field. Along the way, we will also give a brief indication on the possible connection between KAM theorem and phyllotaxis, the leaf arrangement, in botany.
\end{abstract}

\section{What price multimedia?}

I thought I knew something about multimedia. So did Professor C.-K. Hu, chairman of this symposium, who urged me to take up the challenge of giving this talk in a most interesting way so that our intended audience, mostly high school physics teachers, will appreciate the ubiquity of chaotic phenomena. But why me? "Well, you did produce a homemade video [sic., of an understandably poor quality] on chaotic models in some of your other talks, didn't you?"

Okay, so I took up the responsibility, thinking that multimedia was nothing more than adding a few beeps to my old programs to catch the audience's attention. But my optimism quickly went down the drain the moment I spotted the hotshot game DOOM II at a computer show while trying to get a grip of what multimedia was all about - to make a catchy multimedia I need to create a lot of colorful cartoon characters jumping, crawling and squeaking all over the monitor with special FM sound effects when an underlying MIDI music is being played! Was it DOOM II or DOOMed I? I am no longer so sure.

Anyway, following the advice from a sympathetic sales representative I brought home with me a multimedia kit and thus began my adventure. After burning down a $\$ 250$ audio card and several sessions of failed installation of a CD-ROM ,I finally got the 
first sound out from the back of my 486 DX33 computer. Now the hard part: find a software to drive this system. It was then that I realized that my dear FORTRAN had become a prehistoric language no longer supported by our software vendors. I do not C why FORTRAN is inferior. Do you? Now you can imagine what my life was like for the next two months: I shut myself home practicing my (monologue) second foreign language! Lucky me that by the time I felt comfortable working with it nobody was advocating a $\mathrm{D}$ language!

Now that I had all the tools at hand it must be a pleasant experience to actually start the project, right? Wrong again! If you want the computation, the animation, the sounds and the music all come out together in real time, you are unreasonably overloading your poor CPU. Try torturing a DX33, and you will understand why I ended up investing another amount of money on a Pentium...

As you must have guessed by now, the presentation you are about to see is a one-man task; and as such, it is no comparison to the now-familiar fancy multimedia you see on a commercial product. However, it does add a tiny bit of charm and flavor to the beauty of the physics of chaos, to which we now turn.

\section{No room for three bodies}

The study of the infamous "chaos" as we know it today was originated by H. Poincaré in the last century when he was trying to solve the famous three-body problem of celestial mechanics [1]. Although we won't discuss this problem due to lack of time and space, it might be interesting to see how chaos exhibits itself in such a situation. As our animation shows, the trajectory of a tiny satellite moving under the gravitational attraction of the sun is significantly perturbed by the revolving planet which, in effect, contributes to its motion only through a periodic driving force. Indeed, the satellite quickly gets ejected into infinity after several irregular revolutions (Fig. 1), which is not uncommon in many more "serious" studies of problems of this type. In fact, some astronomers think that this mechanism might have to do with the ring formation of Saturn and possibly other planets [2,3].

Somehow three simply is not company in heaven...

\section{The Lorenz equation}

Leaving heavenly bodies aside, we now come to an equally famous, but more down to earth, example: the Lorenz equation

$$
\begin{aligned}
& \dot{X}=10(-X+Y), \\
& \dot{Y}=30 X-Y-X Z, \\
& \dot{Z}=-2.67 Z+X Y .
\end{aligned}
$$




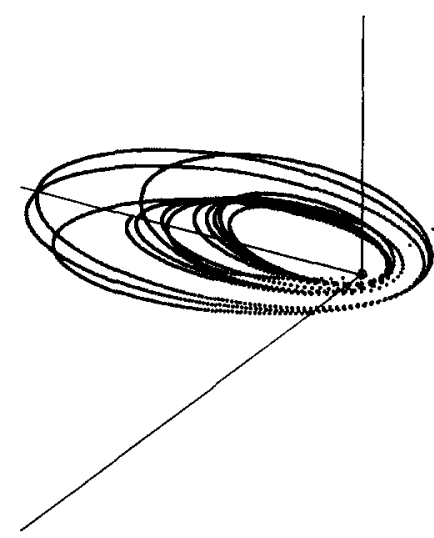

Fig. 1. Trajectory of a satellite moving under the joint influence of the sun and a revolving planet on a circular orbit (not shown). The satellite is ejected to infinity after several irregular revolutions around the sun.

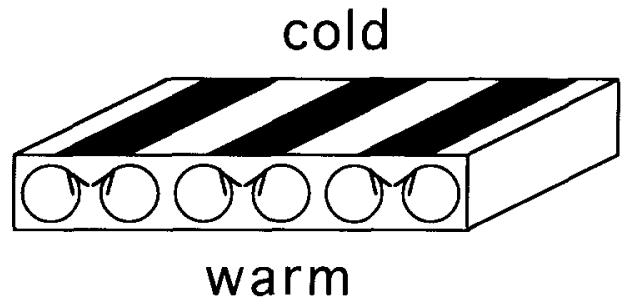

Fig. 2. A shallow container filled with a fluid is uniformly heated from below. When the applied vertical temperature gradient exceeds a certain value convective rolls of definite size will appear.

This set of equations was proposed as a highly simplified model of the thermal convection happening in a thin layer of liquid uniformly heated from below (Fig. 2), such as our atmosphere [4]. Here, $X$ is the amplitude of one mode of the fluid velocity, whereas $Y$ and $Z$ are the amplitudes associated with two modes of the temperature distribution in the fluid. The first equation is $F=m a$ in disguise; it says that the time rate of change of velocity is basically influenced by two factors: the fluid viscosity, thus the $-X$ term, and buoyancy, thus the $+Y$ term. (The other amplitude $Z$ for the temperature does not enter this equation because it represents a mode with a different spatial variation.) The second equation is a recast of the heat transport equation: $30 X$ is there because a faster fluid velocity $X$ will cause a quicker heat exchange between adjacent fluid elements via convection; the appearance of $-Y$ tells us that thermal diffusion has been taken into account. The $-X Z$ term is the non-linear correction to the (linearized) convective heat transport represented by $30 X$. The third equation is still the same heat transport equation, but tailored to show how the other temperature mode, $Z$, evolves in time. That's why it looks very much like a different version of the second equation. (We do not have something like $30 X$ in the third equation for the same reason we do not have a $Z$ in the first: $X$ and $Z$ represent two entirely different spatial configurations.)

To study the time evolution of the Lorenz equation, one can start with a set of initial 


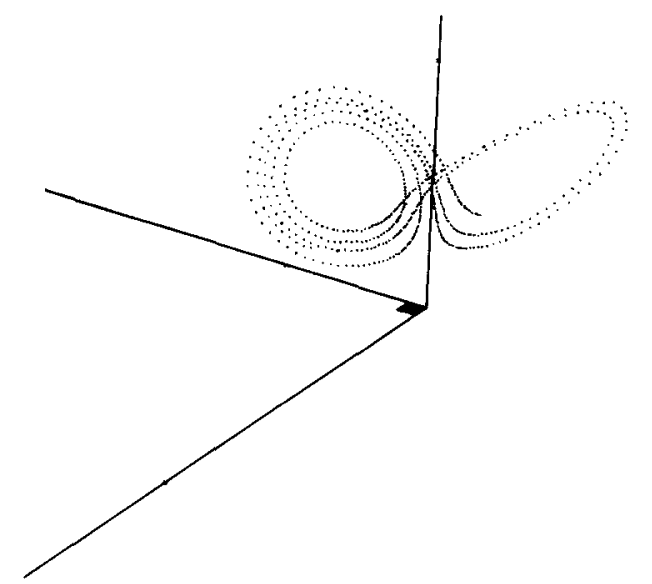

Fig. 3. A test run of the Lorenz equation in the phase space.

data for $X, Y$ and $Z$, plug them into the right-hand side of Eq. (1) to compute their time rate of change. This allows one to predict what they will become for the next moment. Then one can repeat the process again and again to determine its long term behavior. Plotting out the data points in the "phase space", the three-dimensional space formed by $X, Y$ and $Z$, one then obtains a visual representation called phase space trajectory of its evolution. Fig. 3 is a test run using the initial values $X=10, Y=12$ and $Z=15$. Even though we have run only for a short period of time, you can already see that the trajectory seems to have been trapped on two planes intersecting each other at a certain angle, and is constantly switching between them, willy-nilly. Why is that?

The key to understanding this "chaotic" behavior lies in a very beautiful construction due to - you guessed! - Poincaré. Let us draw a disk of radius 10 located at $Z=30$ and see how those points on the disk evolve in time. This is shown in Figs. 4a and $4 \mathrm{~b}$. Clearly, the disk gets squashed in one direction and stretched in another. If we wait patiently in the plane $Z=30$ for these points to cross (and listen to the computer music I have specifically prepared for this occasion so that you won't get bored), then record the intersection points we will get Fig. $4 \mathrm{c}$, which contains two very narrow wedge-like structures. Let the two narrow wedges evolve further, and we have come to Figs. $4 \mathrm{~d}$ and 4e. Fig. $4 \mathrm{e}$ is all the more interesting because it shows that the original continuous time dynamics has been reduced to a discrete mapping, the so-called Poincaré map, which in our case has mapped a circular region into two very narrow wedges intersecting the original domain. For all practical purposes, we may think of the circular disk as having been collapsed into two disjoint line segments since the widths of the two wedges are virtually zero. This is the reason why our numerical simulation seems to suggest that the trajectory lies in two skew planes. Indeed, the map can be schematically represented by Fig. 5, which in effect maps the right half disk into the lower line segment, and the left half disk to the upper line segment. All sources of chaos can be understood using this picture alone!

For simplicity, we shall concentrate on which side (left, $L$, or right, $R$ ) the trajectory 

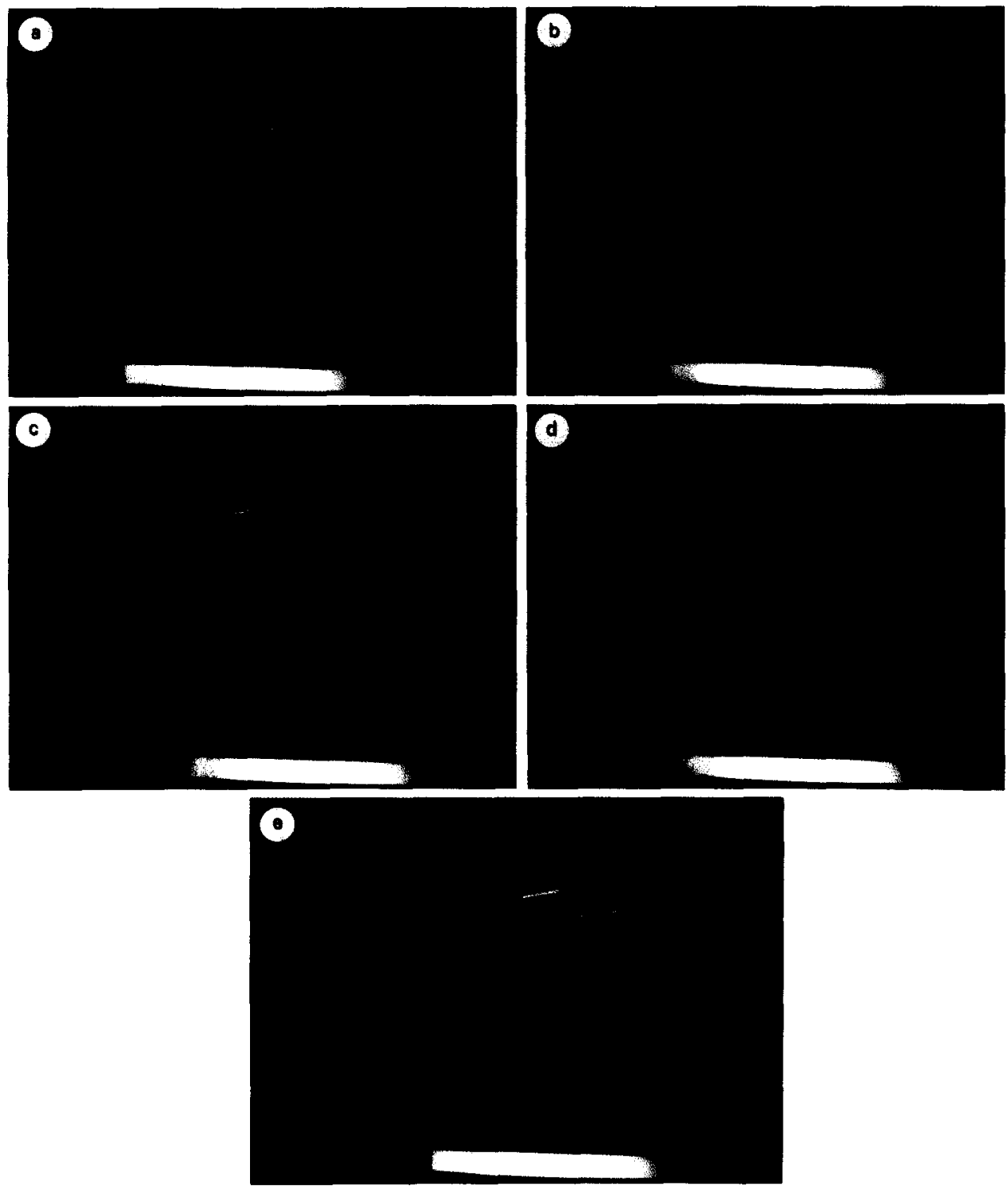

Fig. 4. Evolution of a conveniently chosen disk in the phase space for the Lorenz equation: (a) the starting disk; (b) midway in the evolution; (c) trajectories hit the Poincare section and form two narrow wedges; (d) further evolution down the road; (e) trajectories hit the disk, virtually forming two line-segments.

intersects this "Poincaré section", our plane $Z=30$, each time it crosses it. If it falls on the right-hand side, then it will loop over mainly in the right half space before it hits the Poincaré section next time. But if it falls to the left, then the loop will go around mainly in the left half space. Saying that the trajectory switches chaotically between the left half space and the right half space amounts to saying that a starting point on the Poincaré section is mapped "randomly" under the repeated applications of the Poincaré map, i.e. 


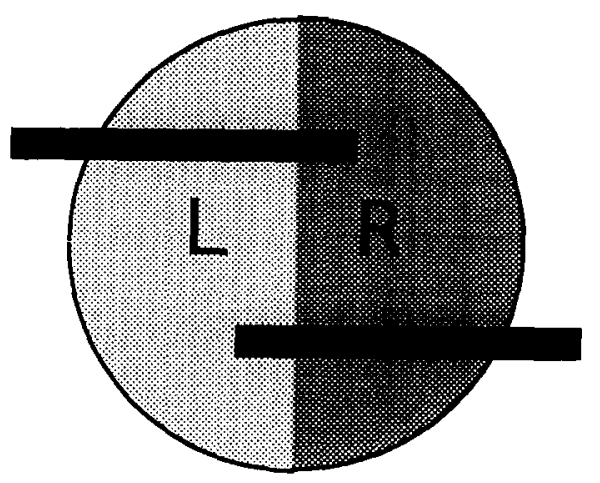

Fig. 5. Schematic drawing of Fig. 4e, showing that the right half disk $(R)$ is mapped down to the lower line segment and the left half disk ( $L$ ) to the upper line segment.

the symbols $L$ and $R$ representing which side the point is on will appear in no apparent order. And this is clearly what is happening here, because both the lower and the upper segments in Fig. 5 extend to both the left and the right Poincare half spaces, meaning that a point originally located on the right-hand side $(R)$ can be brought to either $R$ or $L$ under the Poincaré map, depending crucially on exactly where it was located! Here, the word "exact" cannot be over emphasized, because a slight deviation in the original position can quickly send the trajectory off to something bearing no correlation to our intended trajectory. This fact is coined "sensitive dependence on initial conditions", and is well depicted by Figs. 6a, 6b and 6c.

At this point it is worth summarizing how we get the chaos we observe. Clearly, it is the squashing and the stretching of the trajectories along different directions coupled by the coming back of the trajectories to where they started that have caused the complicated dynamical behavior. Since the latter is usually called "mixing", we may loosely say that the ingredients of chaos are: stretching, squashing, and mixing of phase space points in a finite domain. As will be seen shortly, the same mechanism will resurface for other systems.

\section{A poor man's chaos}

It was rumored that probability theory came about all because a bunch of (poor?) mathematicians were determined to strike it rich at a casino. Too bad physicists were not participating in that early game! Even if any physicist was there, the chances that he would become one of those founders of probability theory might still be very slim because most likely he would be so occupied trying to solve Newton's equation of motion for the roulette or the dices before he placed his bet that he just couldn't afford the time chatting with his mathematician friends about chances, which, presumably, God did not play with anyway.

Assuming our poor physicist friend was granted access to a super fast computer by 
(a)

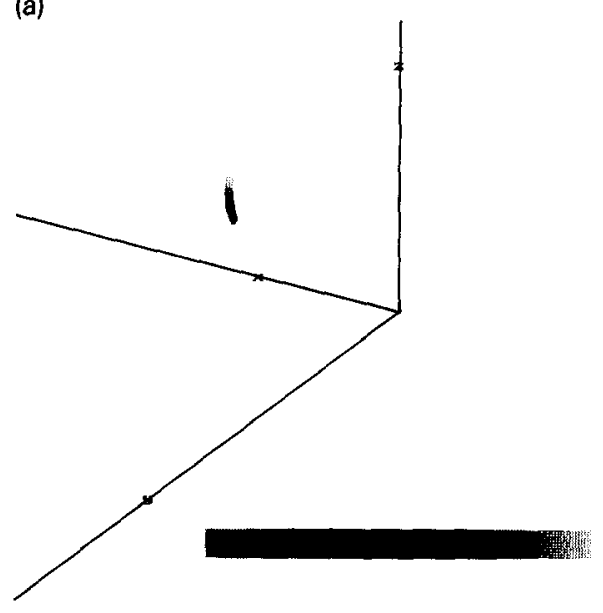

(b)

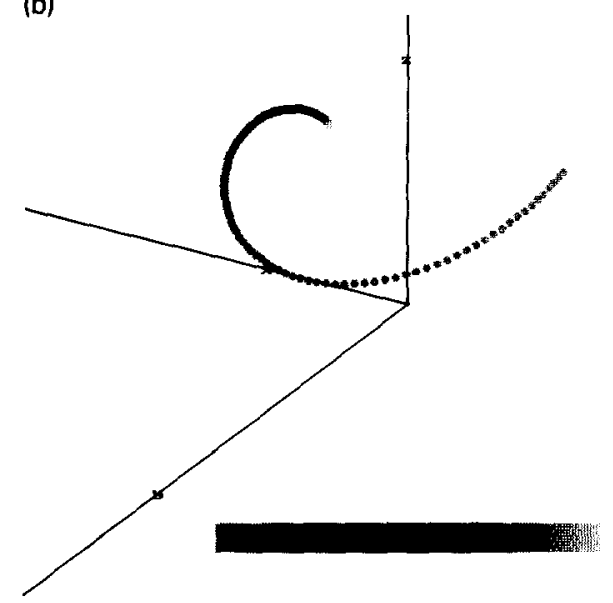

(c)

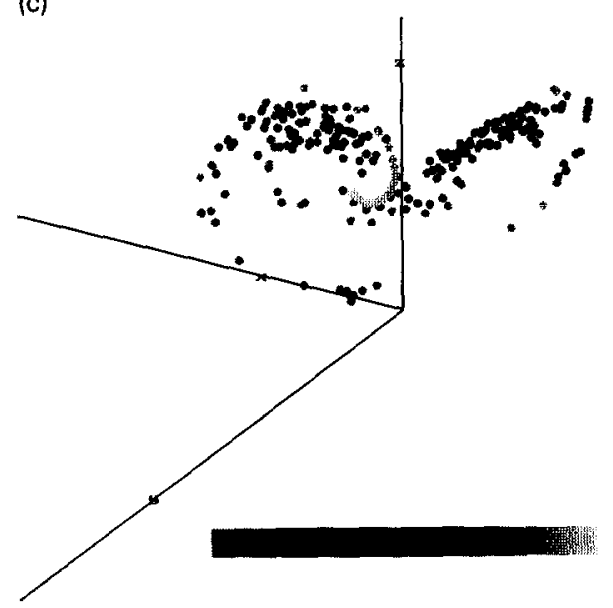

Fig. 6. Sensitive dependence on initial conditions for the Lorenz equation: (a) 248 differently colored, closely packed initial points; (b) trajectories begin to deviate appreciably after a short while; (c) very soon each point moves "randomly" in its own way.

his funding agency on the condition that any big money thus won at a casino must be donated to the agency, would he be able to predict the motion of a tossed dice and helped the agency out of its financial embarrassment?

The answer is yes and no. Yes, because the system is deterministic, meaning that the state of motion for the next moment is completely determined once its present state of motion is known, thus in principle allowing a computer with a truly high accuracy to be able to predict what will happen next, and in the long run. No, because a slightest error in the experimental determination of the present state of the motion of the dice will be so quickly amplified at the next moments that the answer you compute might bear no apparent correlation to the actual state one observes after a short while. (After all, this is what gambling is all about, isn't it?) An old Chinese proverb nicely summarizes this 


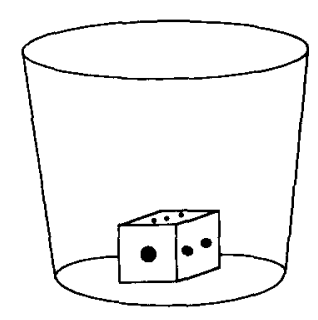

(a)

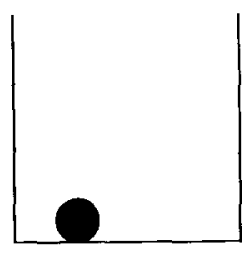

(b)

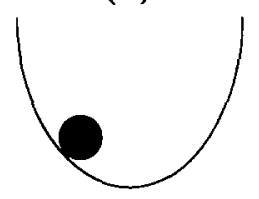

(c)

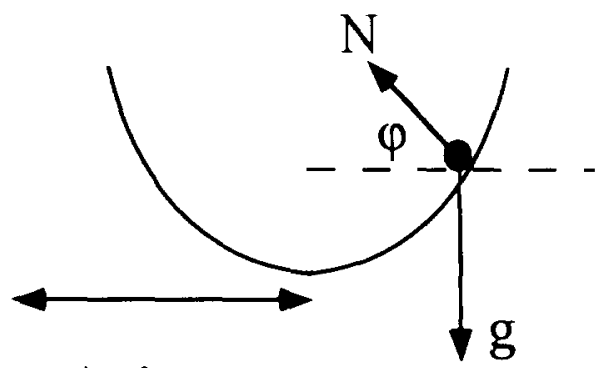

Asin $\omega t$

(d)

Fig. 7. Successive idealization of a gambling model: (a) the original model; (b) dice is replaced by a point particle; (c) hard glass walls are replaced by a smooth parabolic surface; (d) showing the variables related to the model of $(\mathrm{c})$.

fact: "To miss only one millimeter is to cause thousands of miles in error."

To illustrate this point, one might wish to consider the idealized model of having a dice rolling inside a shaking glass and see if any chaotic behavior can be picked up and studied. (See Fig. 7a.) Unfortunately, this whole problem is already too hard for our purpose. So we simply forget about the size of the dice and assume that it is a point particle, hoping that a dice must roll even more chaotically if its idealized counterpart (the particle) has a chaotic trajectory. (See Fig. 7b.) Although this simplified model can be analyzed, it is not convenient for our purpose. For one thing, sudden changes will take place when the particle hits the glass wall. Besides, the particle sometimes might get stuck to the wall, especially when the acceleration is large. (All that I want is something smooth and can be perceived pictorially.) This leads us to a further modification of the model (Fig. $7 \mathrm{c}$ ), which is a point particle of mass $m$ constrained to move on a smooth parabolic surface defined by $y=\frac{1}{2} a x^{2}$ in a local gravitational field $g$, with the confining surface itself executing a horizontal simple harmonic motion described by $x_{\text {center }}=A \sin \omega t$. (In the above, $a$ and $A$ are constant parameters.) The configuration is depicted in Fig. 7d.

We can easily write down the equations of motion for this system:

$$
\begin{aligned}
& \frac{d^{2} x}{d t^{2}}=-\frac{N}{m} \cos \phi+A \omega^{2} \sin \omega t, \\
& \frac{d^{2} y}{d t^{2}}=\frac{N}{m} \sin \phi-g,
\end{aligned}
$$

where $N$ is the (normal) constraint force the surface exerts on the particle, and $\phi$ is the angle between the force $N$ and the horizontal. With the help of $y=\frac{1}{2} a x^{2}$ one may combine the two equations above to yield 


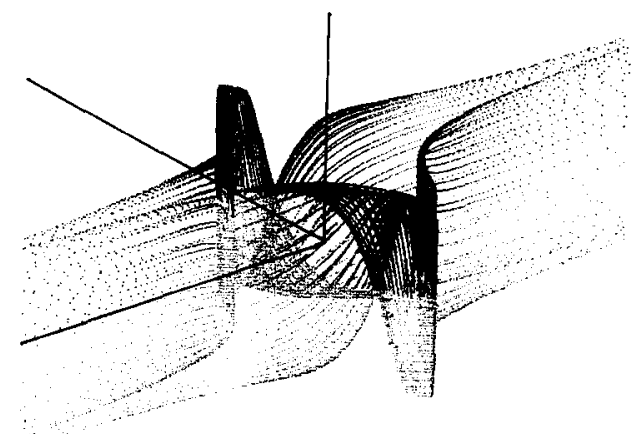

Fig. 8. Phase space trajectory for the gambling model.

$$
\left(1+a^{2} x^{2}\right) \ddot{x}+a^{2} x \dot{x}^{2}+g a x-A \omega^{2} \sin \omega t=0,
$$

which can be further simplified to read

$$
\left(1+X^{2}\right) \frac{d^{2} X}{d T^{2}}+X\left(\frac{d X}{d T}\right)^{2}+\gamma X-\alpha \sin T=0,
$$

where we have tried to non-dimensionalize the variables by setting $X \equiv a x$ and $T \equiv \omega t$, with the two new parameters $\gamma$ representing the local gravitational acceleration and $\alpha$ the acceleration due to the shaking of the surface by some external agent, respectively.

Running the animation for Eq. (2) is easy. All you do is fix a value for the parameters $\alpha$ and $\gamma$, pick an initial value for $X$ and $d X / d T$, then substitute them into Eq. (2) to get $d^{2} X / d T^{2}$. This in effect tells you the velocity $d X / d T$ for the next moment. It is also clear that we can compute $X$ for the next moment too. Then the whole process can be repeated a couple of times till you have reached the time you want. Mathematically, one can conveniently define $Y \equiv d X / d T$ and $Z \equiv \sin T$ then mimic what we did with the Lorenz equation to show its time evolution in the three-dimensional space spanned by $X, Y$ and $Z$, also called the phase space. With $\alpha=5, \gamma=10$ and the initial data $X=15$, $Y=0$ and $Z=0$, the result is shown in Fig. 8. Not too impressive, is it?

Worry not! Even before the invention of computers Poincaré already realized that this may not be the most convenient way to perceive what is going on with a periodically driven system. Poincaré's idea is this: Looking back at Eq. (2) we see that it always reduces to the same form when the time $T$ is advanced by $2 \pi$. This suggests that instead of keeping track of the particle trajectory all the time it might be more illuminating to take a snapshot of its dynamical state at a regular interval of time coinciding with the driving period. For our purpose, we may take the snapshot at an interval of $\pi$, and, lo and behold, we get the neater Fig. 9a.

So far so good. But why do we get a series of chains in Fig. 9a? (We should emphasize that these chains are the accumulated intersections of one single trajectory with the Poincare section, the plane $Z=0$.)

To get a better understanding of this phenomenon, we will look at the case when $\alpha$ is zero, i.e. no external shaking, for some hint. 
(a)

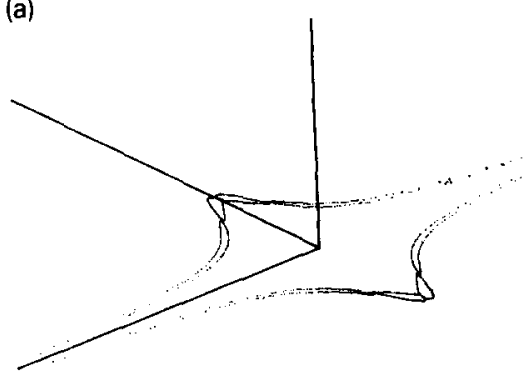

(b)

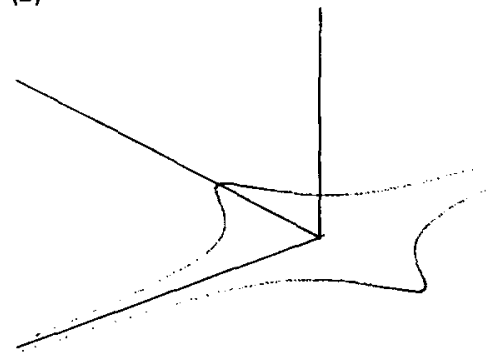

Fig. 9. (a) Chain structures show up for a trajectory repeatedly intersecting the Poincare section when the external shaking for the gambling model is weak. (b) Equal-energy curve for the unperturbed system. It looks like (a) without the chain structures.

When $\alpha$ is set to zero, the total energy $E$ of the system is conserved:

$$
E=\frac{1}{2}\left(1+X^{2}\right)\left(\frac{d X}{d T}\right)^{2}+\frac{\gamma}{2} X^{2}=\text { constant }
$$

The equal-energy curve defined by Eq. (3) is shown in Fig. 9b, which closely resembles Fig. 9a, except that the chain structures are absent.

Even how Fig. $9 \mathrm{~b}$ is obtained is of some interest to us too: In fact, for a given energy $E$ the system will execute a periodic motion with a period depending on $E$. Yet Poincaré is asking us to take a snapshot at an interval of $2 \pi$, which most likely is incommensurate with the particle's intrinsic oscillational period. This implies that starting at any given point on the equal-energy curve your chances of returning to where you started at each moment the snapshot is taken are minimal. You will simply roam over the entire equal-energy curve! Indeed, this is exactly why it is possible to trace out the equal-energy curve (after a long long time) if you simply follow one single trajectory and take snapshots as prescribed.

But suppose you have chosen the energy $E$ in such a way that the intrinsic oscillational period of the particle is $3 \cdot 2 \pi=6 \pi$. This time you are doomed to be bound: Having taken three snapshots you will find yourself back to where you started again. Sorry, no safari! In mathematical terms, every point on the curve is a fixed point if you take a snapshot every $6 \pi$. When this happens we say the external driving and the internal motion are in resonance.

With this carefully selected energy, we now investigate what happens if we turn on the perturbation $\alpha$ by just a little bit. (This means now we are shaking the constraining surface by a small amount.) When $\alpha$ is slightly bigger than zero, the original equalenergy curve will be indented here a little bit and bulges out there a little bit after $2 \pi$, as shown in Fig. 10. Note that point $P^{\prime}$, one of the intersections of the original curve with its image under the Poincaré map, must be very close to its preimage $P$, because $P$ is necessarily a fixed point by our construction when the perturbation $\alpha$ is zero.

So, here is what we have put ourselves in: Point $P$ is mapped back to a slightly shifted point $P^{\prime}$ on the original curve. If we now increase the energy $E$ by a small amount, 


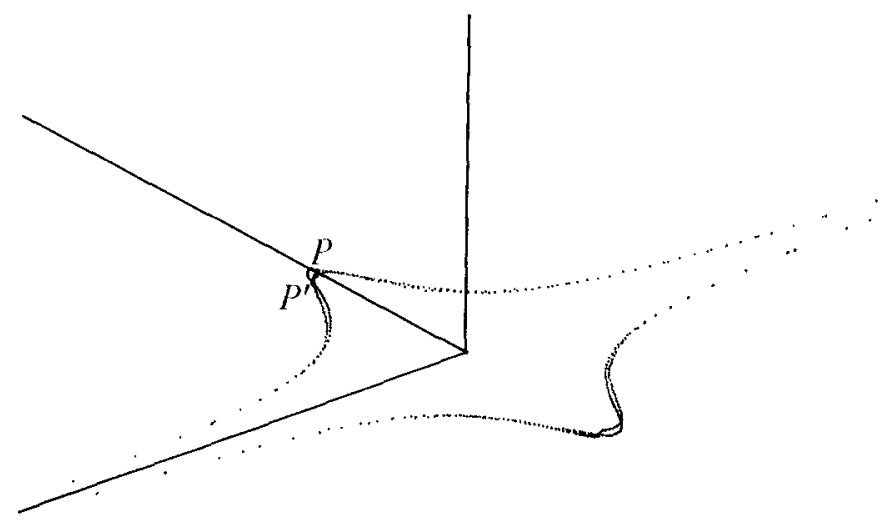

Fig. 10. The Poincare map of the equal-energy curve of Fig. $9 \mathrm{~b}$. The new curve intersects the old one at several points. The preimage of $P^{\prime}$ is $P$.

chances are $P^{\prime}$ will move closer to $P$. (If the opposite is true, then you should decrease the energy instead.) Increasing (or decreasing) the energy further, eventually we can make the two points overlap, in which case we have found a fixed point even when $\alpha$ is non-zero! What we just described is a crude roundup of the famous Poincaré-Birkhoff theorem.

Once we have a fixed point, we can begin investigating particle trajectories which happen to fall close to it. In particular, if a fixed point is stable, meaning that starting at any point close to that fixed point one simply gets trapped and cannot escape to somewhere far and away, then the repeated applications of the Poincaré map will usually yield a closed curve for the same reason we could roam along the equal-energy curve to actually trace out its shape when $\alpha$ is zero. This explains why we see several chains in Fig. 9a: Somewhere in the middle of each chain is a certain stable fixed point.

But what about the unstable fixed points? Ah, things become much more exciting when something is unstable. (Note: CIA is not the only one in the world who always gets excited more by instabilities.) Near an unstable fixed point, most points will eventually be driven away under the repeated applications of the Poincaré map. But you can find one direction along which the points are expelled at the fastest speed. This is called the unstable manifold. Likewise, one can find a direction along which points are actually attracted to the fixed point. This is called the stable manifold. It is harder to observe this stable manifold near an unstable fixed point because a slightest deviation from it will send a point off track and kick it off in the long (or, rather, short?) run. This is shown schematically in Fig. 11a. Let us assume that the stable manifold $W_{1}$ intersects the unstable manifold $W_{2}$ at a point $Q$. Since $Q$ is located on $W_{1}$, it is going to be brought nearer and nearer the unstable fixed point $P$ under the Poincare map. This is shown in Fig. $11 \mathrm{~b}$. But since $Q$ also happens to sit on $W_{2}$, we know that, by continuity argument, near the images $Q_{1}, Q_{2}, \ldots$ of $Q$ must also lie a portion of $W_{2}$. This implies that $W_{2}$ must deform a great deal near $P$, because things are stretched in one direction (the original $W_{2}$ direction) and compressed in another (the original $W_{1}$ direction). As 
(a)

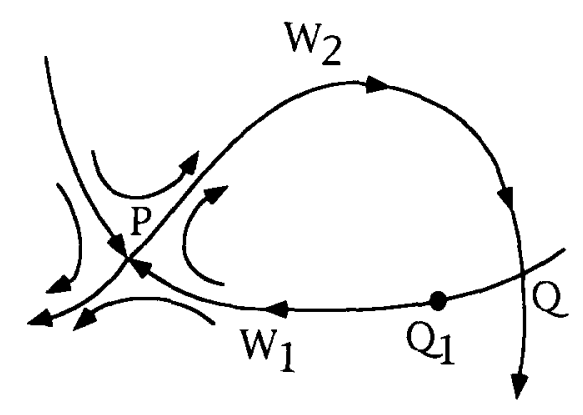

(b)

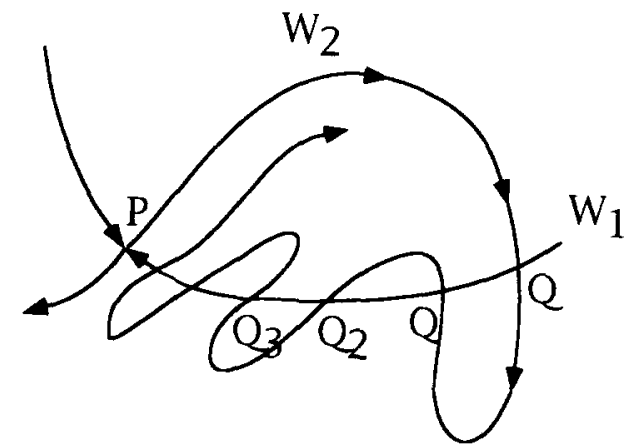

Fig. 11. (a) The stable manifold $\left(W_{1}\right)$ intersects the unstable manifold $\left(W_{2}\right)$ at point $Q . Q$ will be brought to some point $Q_{1}$ nearer the unstable fixed point $P$ under the Poincaré map. (b) $Q$ is successively mapped to $Q_{2}, Q_{3}, \ldots$, showing that $W_{2}$ must also come back near $P$, but highly deformed.

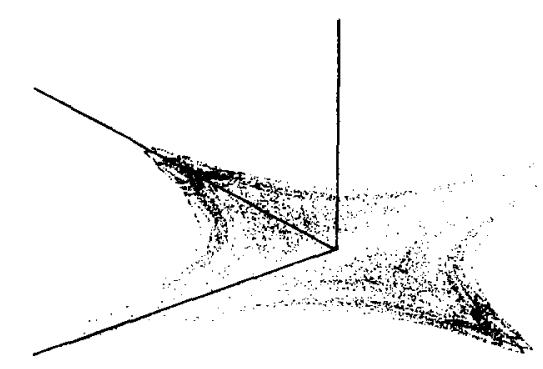

Fig. 12. Trajectory on the Poincaré section scatters all over the plane, demonstrating that it is indeed chaotic.

you can see, most points starting out near $P$ will be quickly kicked off the entangled region and only to find itself sucked in near $P$ again after a short while. What do we have then? Chaos, of course!

At this point it seems appropriate to emphasize once again that the reason we have chaos here is that (1) stretching (in the $W_{2}$ direction) and compression (in the $W_{1}$ direction) coexist in the region, and (2) mixing does occur (because $W_{2}$ actually crawls back near the fixed point along the direction of the stable manifold, $W_{1}$, though highly deformed, of course).

Just to give you an idea of how significant the external shaking should be in order to generate an appreciable amount of chaos, we show in Fig. 12 the case with $\alpha=30$, $\gamma=10$, and the initial data $X=10, Y=0$ and $Z=0$. As you can see, the trajectory is scattered out in a large region with no obvious regularity at all. Because $\alpha / \gamma \quad(=3)$ is a relative measure of how fast one is shaking the system, we see that the numerical experiment does suggest that a mild shaking is enough to generate chaos. Though possibly a disappointment to that physicist friend of ours who was trying to pay his mentor back, the conclusion is quite a relief to most of us who only visit Vegas for fun and not to be ripped off. (If a physicist could predict the rolling of a dice, a gambler would have collected enough fortune to buy out the whole world long ago.) 


\section{From Saturn to Venus to sunflowers}

The toy model we discussed above and the three-body problem we mentioned before both share one significant feature: they are "Hamiltonian systems", meaning that when applying Newton's law $F=m a$ the force $F$ is derivable from a certain potential. Although the cause of chaos for the Lorenz equation is very similar to that for these two Hamiltonian systems (remember the stretching, the squashing, and the mixing?), the former is dissipative and non-Hamiltonian. Because of their special structure, certain Hamiltonian systems such as ours enjoy a particularly interesting property: they possess a number of constants of the motion just enough to completely specify their motion, provided we can identify a "small" perturbation and shut it off. In technical terms, they are "completely integrable" before perturbation. For example, the "small" perturbation in our gambling model is the horizontal shaking - turning it off, and we have the conservation of energy, which is just enough for us to determine the time evolution of the particle.

Turning back to our toy model, the careful audience would notice that we had deliberately left out one important case: we said that near a resonant trajectory certain chaotic behavior was expected to happen, but what about those non-resonant trajectories which have a ratio (intrinsic frequency/external driving frequency) $\neq$ simple rational numbers?

Our intuition tells us that when the internal motion and the external driving are very much out of phase, the driving mechanism simply cannot cause any qualitative change to the motion of the system, because its influences will eventually be averaged out so that one does not perceive appreciably any of the cumulative effects. This is true, and has been known as the KAM theorem after those great minds - Kolmogorov, Arnold and Moser - who had the insight to proving it [5,6]. What KAM says is basically this: regular motions will survive the perturbation if the ratio of the intrinsic frequency to the external driving frequency is very irrational.

Very irrational? Boy, hard to imagine this would come out of the mouth of those very rational mathematicians. Without going into the details, let me just mention that a really very irrational number is the naive-looking golden mean $\phi \equiv \frac{\sqrt{5}-1}{2}$, which some observed to be exactly the enviable figure Venus possessed: divide the length of her navel to the soles of her feet by her body height, and you would get this golden mean [7]!

If you think this is rubbish, wait till you hear this. Many plants actually are numbersuperstitious: They just love the golden mean more than any other numbers! Hummmm...

Fig. 13a is a picture of a sunflower, which probably is one of the most well-known of those plants which seem to have incorporated in their growth mechanism the golden mean: the angle between two consecutively budding seeds (or florets or leaflets, as the case may be) seems to be very close to $2 \pi$ times the golden mean $\phi$. From time to time you might hear someone say that the numbers of spirals you see on a sunflower head are always some of the Fibonacci numbers, which, as you might recall, are 1, 1, $2,3,5,8,13,21,34,55, \ldots$, i.e. the next in the sequence is the sum of the previous two numbers. (See Fig. 13b.) I would like to remind you that this feature is a direct 


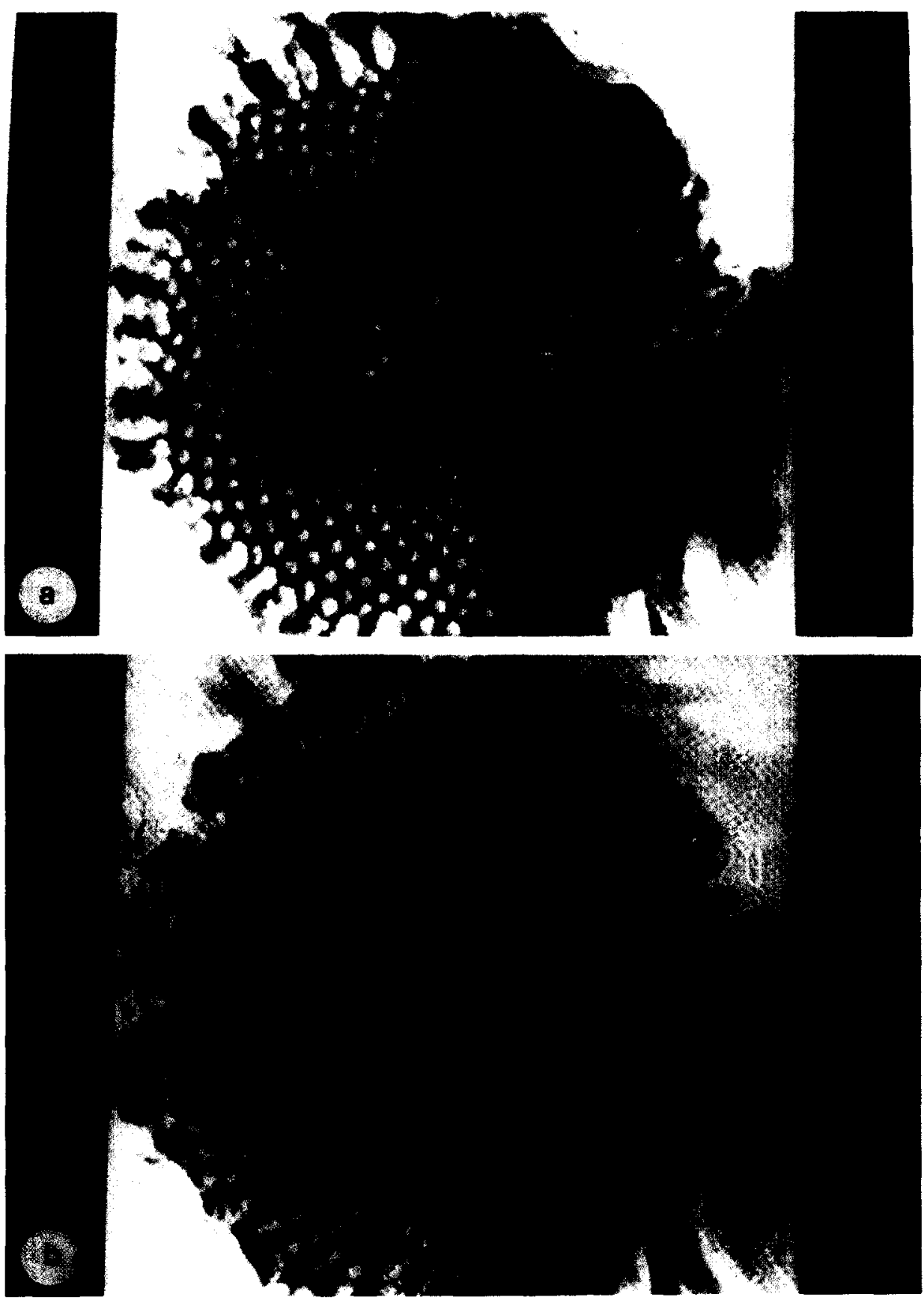

Fig. 13. Pictures of a sunflower head: (a) the original; (b) hand-traced spiral structures revealing the Fibonacci numbers 34 and 55 . 
consequence of the fact that the seeds on the sunflower head come out separated by the angle $2 \pi \phi$, and is not linked to some mystic number worship.

To explain how $2 \pi \phi$, the golden angle, can appear naturally out of a reasonable theory, scientists have proposed several models [8-13], one of which is of the reaction-diffusion type. Loosely speaking, in a reaction-diffusion model one supposes that the growth of a leaflet is activated and/or inhibited by several chemicals, which themselves are undergoing complex reactions while diffusing into their surroundings. A budding leaflet will send out a strong concentration of inhibitors to prevent the accidental budding of another leaf near it. Only when a place in the growing region has an inhibitor concentration lower than a certain threshold does a new leaf begin to grow there. Hence, to determine the position of the next budding leaflet all we have to do is track down the position of the lowest inhibitor concentration in space at every moment. Our study shows that under certain approximations the position of the lowest inhibitor concentration moves in space in such a way that it satisfies Newton's $F=m a$, with an $F$ derivable from a potential. In other words, it is a Hamiltonian system with a periodic kicking, just like our previous gambling model! [14] (The periodic kicking for this case is caused by the periodic budding of new leaves.) Because the most irrational number $\phi$ usually corresponds to the last regular (periodic) orbit a perturbation destroys in an otherwise completely integrable Hamiltonian system, the ubiquitous appearance of the golden angle in plants seems to suggest that the internal physico-chemical environment inside those plants exhibiting the golden angle might have been fine-tuned either by accident or by evolution to admit such occurrence. (It is conceivable that evolution might play an important role in this respect because one can easily show that leaf arrangements related to very irrational angles such as the golden angle tend to give the most uniform distribution of leaves [15].) Since the details of our investigation of this problem are under preparation, we will give a more thorough report on this aspect somewhere else.

To summarize: Although chaos is now known to be a fairly common phenomenon, regular motions do not simply take their bows and step down. Instead, they can coexist quite happily with chaos under circumstances. One manifestation of this is the KAM theorem dealing with perturbed completely integrable Hamiltonian systems. Nature permitting, one implication of the KAM theorem that the most robust periodic orbits could be those having a frequency ratio of $\phi$, the golden mean, with respect to the external driving might have something to do with the fact that certain plants exhibit the golden angle in their leaf arrangement.

\section{Conclusion?}

A short talk like this can never do justice to the huge body of knowledge scientists have acquired on any topic. This is especially true with the exploding field of chaos. The fact that chaos usually occurs when stretching, squashing and mixing act together has been well-known for quite a long time, though $I$ have sneaked in our recent finding of the connection between implications of the KAM theorem and the leaf arrangement 
of some plants. To restrict ourselves to the easiest aspects of chaos, I have left the even more fascinating subject of spatio-temporal chaos totally untouched. I hope my audience will forgive me for such a deliberate negligence. If we are to draw any conclusion from such an ill-nourished presentation, I would only say three things:

- Chaos is usually related to the stretching, squashing, and mixing of points in the phase space. (Okay, you already knew that.)

- The Poincaré map can be a handy tool when it comes down to actually analyzing a chaotic system. (Oh, yes, you knew that too.)

- Multimedia is the trend for more illuminating education. Real experts should devote their time working out such a project for the benefits of our students, and me!

\section{Acknowledgements}

I thank Professor C.K. Hu for the invitation and for entrusting me with the designing of the poster for this symposium. Constant encouragement for this project from Dr. C.K. Chan at the Institute of Physics of the Academia Sinica is greatly appreciated. Suggestions from Dr. Y.K. Hu concerning how part of the graphics could be rendered had helped me a lot. Special thanks also go to Professor S.S. Hsiao at the Department of Mechanical Engineering of National Central University and to Dr. C.K. Chan for their kindness in preparing experimental film clips specifically for this talk.

\section{References}

[1] H. Poincaré, New Methods of Celestial Mechanics, Vols. 1-3, ed. D.L. Goroff (AIP, New York, 1993 ).

12| J. Wisdom, Astron. J. 87 (1982) 577.

13| M. Duncan, T. Quinn and S. Tremaine, Icarus 82 (1989) 402.

14| E.N. Lorenz, J. Atmos, Sci. 20 (1963) 130.

I5] V.I. Anold, Russ. Math. Surveys 18 (1963) 5.

[6] J. Moser, Math. Ann. 169 (1967) 163.

17| T.A. Cook, The Curves of Life, Dover, New York (1979) 426.

18| I. Adler, J. Theor. Biol. 45 (1974) 1.

19| J.H.M. Thornley, Ann. Bot. 39 (1975) 491.

| 10| G.J. Mitchison, Science 196 (1977) 270.

[11] R.V. Jean, J. Theor. Biol. 71 (1978) 639.

| 12 | D.W. Roberts, J. Theor. Biol. 108 (1984) 481.

| $13 \mid$ G.P. Bernasconi, Physica D 70 (1994) 90.

$114 \mid$ Y.-Y. Chen, in preparation.

| 15| J.N. Ridley, Math. Biosciences 58 (1982) 129. 